\title{
Broadband Adaptive RCS Computation through Characteristic Basis Function Method
}

\author{
Guohua Wang and Yufa Sun \\ Key Laboratory of Intelligent Computing \& Signal Processing, Ministry of Education, \\ Anhui University, No. 111 Jiu Long Road, Hefei 230601, China \\ Correspondence should be addressed to Guohua Wang; wangguohua_2014@163.com
}

Received 23 February 2014; Accepted 12 June 2014; Published 21 August 2014

Academic Editor: Igor Djurović

Copyright (c) 2014 G. Wang and Y. Sun. This is an open access article distributed under the Creative Commons Attribution License, which permits unrestricted use, distribution, and reproduction in any medium, provided the original work is properly cited.

\begin{abstract}
A broadband radar cross section (RCS) calculation approach is proposed based on the characteristic basis function method (CBFM). In the proposed approach, the desired arbitrary frequency band is adaptively divided into multiple subband in consideration of the characteristic basis functions (CBFs) number, which can reduce the universal characteristic basis functions (UCBFs) numbers after singular value decomposition (SVD) procedure at lower subfrequency band. Then, the desired RCS data can be obtained by splicing the RCS data in each subfrequency band. Numerical results demonstrate that the proposed method achieve a high accuracy and efficiency over a wide frequency range.
\end{abstract}

\section{Introduction}

With the development of detection technology, the topic of efficient and accurate solution of the electromagnetic scattering problems over a broad frequency band has drawn increasing attention. The broadband electromagnetic scattering is very important in many fields such as modern radar target recognizing, microwave imaging, and microwave remote sensing. We can obtain more comprehensive information from broadband RCS data, such as volume information, contour information, details information, and so on, which has important significance in stealth and antistealth fields.

The traditional method of moments (MoM) can be applied for accurate solution of broadband electromagnetic problems. To obtain the RCS data over a wide band range using $\mathrm{MoM}$, one has to repeat the calculation processes at each frequency point over the desired band, and this is inefficient when the object size as well as the number of frequency sampling points is large. Recently, several techniques have been proposed to circumvent this problem. In [1], the model-based parameter estimation based on rational function approximation is used to reduce the number of frequency points at which solutions or samples are required in broadband RCS calculation. In the papers [2, 3], asymptotic waveform estimation (AWE) technique has been successfully applied to obtain the broadband RCS data. But AWE is accurate only around the frequency of expansion; its accuracy deteriorates beyond a certain bandwidth. In [4], the impedance matrix is computed at relatively large frequency intervals and then interpolated to approximate its values. However, all the above techniques need resort to iterative methods and thus the target's monostatic RCS cannot be calculated very efficiently. In the papers [5-7], an algorithm that utilizes the characteristic basis function method (CBFM) $[8,9]$ has been presented for computing the broadband RCS data. This approach takes the characteristic basis functions (CBFs) at the highest frequency after singular value decomposition (SVD) procedure as universal characteristic basis functions (UCBFs). However, it should be noted that the number of UCBFs is higher than necessary and the computational complexity will be increased when applying the UCBFs to the lower frequency points. In many practical application of engineering, one often need to analyze the electromagnetic scattering characteristics over arbitrary frequency band. In this paper, we introduce a new adaptive construction method that can calculate the broadband RCS over any given frequency band. This method leads to relatively small number of UCBFs at lower subfrequency band, so the fill process of the impedance matrix has been sped up and the memory requirements have been reduced significantly. 
The rest of the paper is organized as follows: the detail implementation process of the CBFM is presented in Section 2. Section 3 derives the adaptive construction method of the UCBFs. In Section 4, numerical results for two test targets are presented and compared with the UCBF method over the bandwidth. Conclusions are drawn in Section 5 .

\section{Universal Characteristic Basis Functions Method}

The algorithm begins by modeling the target at the highest frequency point of desired frequency band, using conventional MoM formulation, the electric field integral equation (EFIE) can be transformed into a dense, complex linear system of the form (1)

$$
\mathbf{Z} \cdot \mathbf{J}=\mathbf{V}
$$

where $\mathbf{Z}$ represents the generalized impedance matrix of dimension $N \times N, \mathbf{J}$ and $\mathbf{V}$ are vectors, and $N$ is the number of unknowns.

In this paper, we apply the CBFM based on SVD to generate the characteristic basis functions (CBFs) by a series of illuminating wave. Let us suppose that we calculate the CBFs at the highest frequency point $f_{h}$, the CBFM-SVD begins with dividing the geometry of the object into $M$ blocks, characterizing these parts alone by using CBFs, which represent not only the self-interactions but also account for the mutual coupling effects between the self-blocks and the remaining blocks. We consider totally $N_{\mathrm{PW}}$ plane waves, for examples $N_{\mathrm{PW}}=400$, here, the number of plane wave angles $N_{\text {PW }}$ is deliberately overestimated. The CBFs on the subblock $i$ are constructed by the equation

$$
\mathbf{Z}_{i i}\left(f_{h}\right) \mathbf{J}_{i}^{\mathrm{CBF}}=\mathbf{V}_{i}^{\mathrm{MPW}}, \quad(i=1,2, \ldots, M),
$$

where, $\mathrm{J}_{i}^{\mathrm{CBF}}$ represents a $M_{i} \times N_{\mathrm{PW}}$ matrix containing the CBFs stored column-wise; $V_{i}^{\mathrm{MPW}}$ represents a $M_{i} \times N_{\mathrm{PW}}$ matrix containing the excitation vector used to illuminate the subblock $i$; and $\mathbf{Z}_{i i}$ is a $M_{i} \times M_{i}$ impedance matrix of the subblock $i, M_{i}$ is the number of unknowns in the subblock $i$. Typically, the number of plane waves we have used to generate the CBFs would exceed the number of degrees of freedom (DoFs) associated with the block, to eliminate the redundant information in $\mathrm{J}_{i}^{\mathrm{CBF}}$ caused by overestimation, an orthogonalization procedure based on SVD method is used to reduce the final number of CBFs, that also serves to improve the condition number of the reduced matrix.

We arranged the CBFs obtained from multiple incident conditions in a rectangular matrix and retained only those whose singular values are above a certain threshold (typically chosen to be $1.0 \mathrm{e}-3$, associated with the first and the most influential singular values). For the sake of simplicity, we assume that all of the blocks contain the same number of CBFs after SVD for each of the blocks. The solution to the entire problem is then expressed as a linear combination of the CBFs as follows:

$$
\mathbf{J}=\sum_{m=1}^{M} \sum_{k=1}^{K} \alpha_{m}^{k}\left(f_{h}\right) J_{m}^{\mathrm{CBF}_{k}} .
$$

In (3), $J_{m}^{\mathrm{CBF}_{k}}$ represents the $k$ th CBFs of the block $m$ after SVD. $\alpha_{m}^{k}(f)$ represents the unknown weight coefficients and the solution is obtained at frequency $f_{h}$. Substitute formula (3) into MoM equation (1), we can generate the $\mathrm{KM} \times \mathrm{KM}$ reduced matrix

$$
\mathbf{Z}^{\mathbf{R}}(f) \cdot \boldsymbol{\alpha}(f)=\mathbf{V}^{\mathbf{R}}(f)
$$

where, $\mathbf{Z}^{\mathbf{R}}(f)$ represents the reduced impedance matrix of dimension $\mathrm{KM} \times \mathrm{KM}$, each element of which can be expressed as

$$
Z_{i j}^{R}(f)=\mathbf{J}^{\mathbf{T}} \cdot \mathbf{Z}_{i j}(f) \cdot \mathbf{J} \quad i, j<M
$$

the elements of $\mathbf{V}^{\mathbf{R}}(f)$ can be expressed as

$$
V_{i}^{R}(f)=\mathbf{J}^{\mathrm{T}} \cdot \mathbf{V}_{i}(f) \quad i<M .
$$

Finally, the reduced system (4) is solved for the unknown weight coefficients $\alpha_{m}^{k}(f)$ and the CBFs are obtained at frequency $f_{h}$. If these CBFs can be used in the whole frequency band, then we called them the UCBFs. The UCBFs capture the electromagnetic behaviour of the lower frequencies as well, enable one to solve the scattering for any frequency sample in the band without going through the time-consuming process to generate the CBFs anew.

\section{Adaptive Generation of UCBFs}

In the proposed approach, the any given frequency band is adaptively divided into multiple subbands in consideration of the UCBFs number, then we can calculate the RCS utilizing the UCBFs generated at the highest frequency point in each subband, and the whole RCS data can be obtained by splicing the RCS data of each frequency subband. For a given arbitrary frequency band, the adaptive method named after adaptive UCBF method (Adaptive UCBFM) is described as follows:

(1) setting the initial frequency point $f_{L}=f_{\min }$ and $f_{H}=$ $f_{\max }$

(2) generating the UCBFs in frequency point $f_{H}$ and $f_{L}$, the total UCBFs number of all subblocks is

$$
\begin{gathered}
K_{H}=K_{H 1}+K_{H 2}+\cdots K_{H M} \\
K_{L}=K_{L 1}+K_{L 2}+\cdots K_{L M} .
\end{gathered}
$$

In (7), $K_{H 1}, K_{H 2}, \ldots, K_{H M}$ represent the UCBFs number of subblock $1,2 \cdots M$ at frequency point $f_{H}$, and $K_{L 1}, K_{L 2}, \ldots, K_{L M}$ represent the ones at frequency point $f_{L}$. Given a threshold level $\varepsilon(0<\varepsilon \leq$ 1 , typically chosen to be 0.5$)$, if $K_{L} / K_{H}>\varepsilon$, the RCS over the whole frequency band can be calculated using the UCBFs generating at $f_{H}$ repeatedly, else take $f_{\text {mid }}=\left(f_{H}+f_{L}\right) / 2$, repeat step 2) in $\left[f_{L}, f_{\text {mid }}\right]$ and $\left[f_{\text {mid }}, f_{H}\right]$ until $K_{L} / K_{H}>\varepsilon$;

(3) getting the wide RCS data over a given arbitrary frequency band $\left[f_{\min }, f_{\max }\right]$ by splicing the RCS data in each frequency subband. 
TABLE 1: Number of the UCBFs of the plate.

\begin{tabular}{lcccc}
\hline $\begin{array}{l}\text { Frequency subband } \\
(\mathrm{MHz})\end{array}$ & Block 1 & Block 2 & Block 3 & Block 4 \\
\hline $300-975$ & 20 & 21 & 13 & 14 \\
$975-1650$ & 30 & 32 & 32 & 30 \\
$1650-3000$ & 52 & 53 & 54 & 51 \\
\hline
\end{tabular}

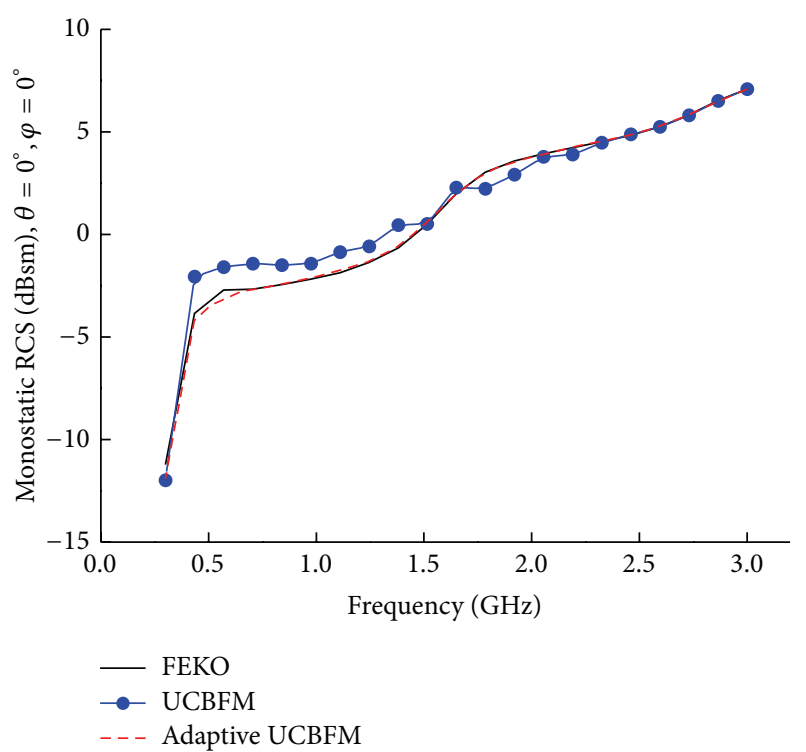

FIgURE 1: Broadband monostatic RCS of PEC plate.

\section{Numerical Results}

In order to validate the accuracy and efficiency of the proposed approach; we present the results for the problem of scattering by a PEC plate and a NASA almond. A conventional triangular patch segmentation and RWG basis functions [10] is employed. The results have been compared with those derived by using UCBF and MoM (FEKO). All the results are computed on an Intel(R) Core (TM)2 Duo PC with $3.40 \mathrm{GHz}$ processor and $4 \mathrm{~GB}$ RAM, only one core is used. The percentage error of Adaptive UCBFM is defined as

$$
\sqrt{\frac{\|\mathbf{V}-\mathbf{Z} \cdot \mathbf{J}\|}{\|\mathbf{V}\|}} \times 100 \%
$$

As a validation example, let us start by considering the problem of scattering by a PEC plate over a frequency range of $0.3 \mathrm{GHz}$ to $3 \mathrm{GHz}$, which is $25 \mathrm{~cm} \times 25 \mathrm{~cm}$ in size located on the xoy plane. The geometry is divided into 1176 triangular patches with an average length of $\lambda / 10$ at $3 \mathrm{GHz}$, and this gives rise to 1764 unknowns. The geometry is then subdivided into 4 blocks, each block has been extended by $\Delta=0.2 \lambda$ in all directions and analyzed for a spectrum of plane waves incident from $0^{\circ} \leq \theta \leq 180^{\circ}$ and $0^{\circ} \leq \varphi \leq 360^{\circ}$, with $N_{\theta}=20$, $N_{\varphi}=10$. The UCBFs after SVD retained at each subband were shown in Table 1.

The object is then excited by a normally incident $(\theta=$ $0^{\circ}, \phi=0^{\circ}$, polarization angle $=90^{\circ}$ ) plane wave. Then

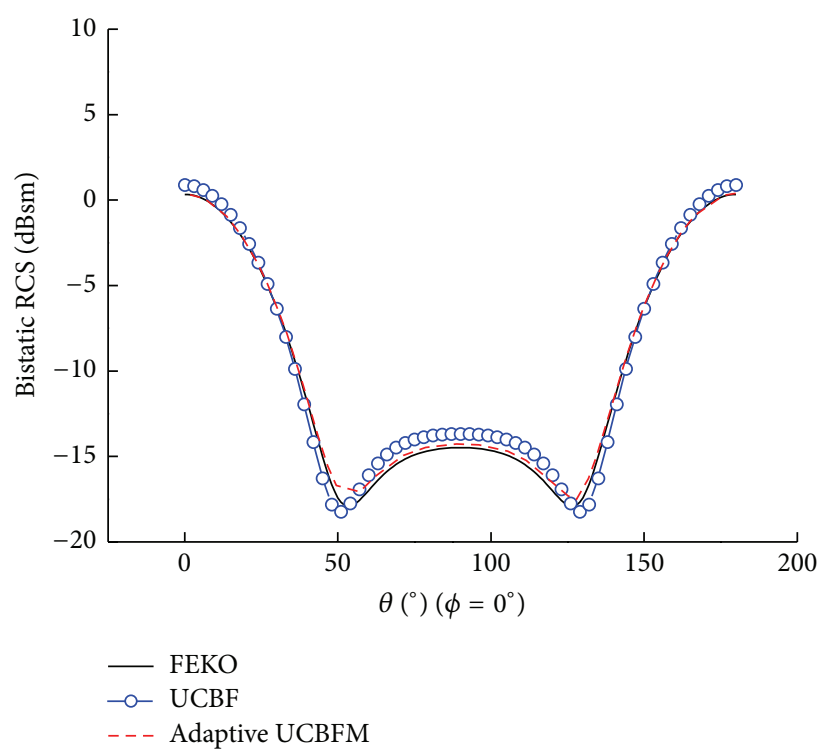

FIgURE 2: Bistatic RCS of PEC plate at $1.5 \mathrm{GHz}$.

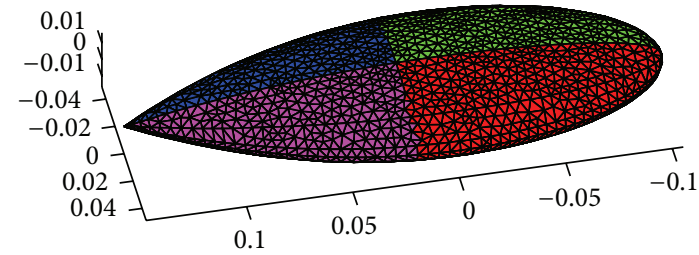

FIgURE 3: Geometry of NASA PEC almond.

TABLE 2: Number of the UCBFs of the almond.

\begin{tabular}{lcccc}
\hline $\begin{array}{l}\text { Frequency subband } \\
(\mathrm{GHz})\end{array}$ & Block 1 & Block 2 & Block 3 & Block 4 \\
\hline $0.3-1.0$ & 13 & 17 & 16 & 11 \\
$1.0-1.7$ & 19 & 27 & 21 & 14 \\
$1.7-2.4$ & 30 & 37 & 41 & 25 \\
$2.4-3.1$ & 29 & 68 & 46 & 24 \\
\hline
\end{tabular}

the monostatic RCS results (21 frequency sampling points) using the method proposed in this paper over the bandwidth are shown in Figure 1. The bistatic RCS calculated for $\mathrm{HH}$ polarization at $1.5 \mathrm{GHz}$ is shown in Figure 2. The results obtained by using the commercial MoM (FEKO) and the $\mathrm{UCBF}$ approaches are also included in Figure 2 for the sake of comparison.

From Figures 1 and 2, we can see that the Adaptive UCBFM has a better match with classic MoM (FEKO) than UCBF without a compromise of the accuracy.

To further illustrate the accuracy of the method, we also present the results for the problem of scattering by a PEC almond shown in Figure 3, which is described in [11].

The total length of almond is $25 \mathrm{~cm}$ along axis $x$. The discretisation in triangular patches is carried out at $3.1 \mathrm{GHz}$ with a mean edge length of $\lambda / 10$, this leads to a number of 
TABLE 3: CPU time and memory requirements.

\begin{tabular}{lcccc}
\hline Example target & Method & CPU time/s & Memory requirements/MB & Percentage error \\
\hline \multirow{3}{*}{ Plate } & MoM & 326 & 23.7 & $/$ \\
& UCBF & 188 & 8.65 & $1.5 \%$ \\
\hline \multirow{3}{*}{ Almond } & Adaptive UCBFM & 92 & 5.34 & $0.2 \%$ \\
& MoM & 1056 & 130.06 & $/$ \\
& UCBF & 659 & 36.25 & $2.4 \%$ \\
\hline
\end{tabular}

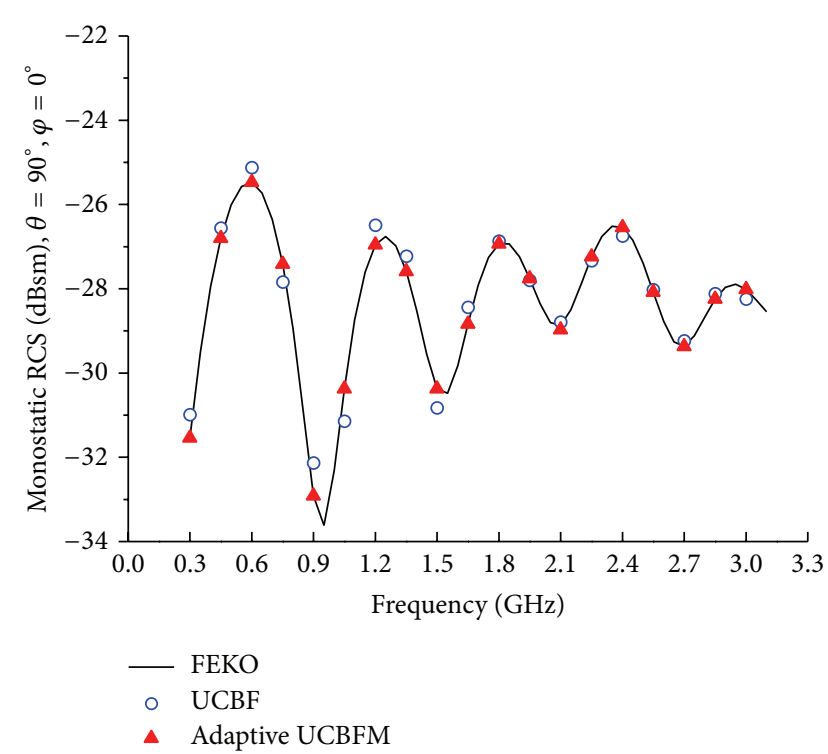

FIGURE 4: Broadband monostatic RCS of almond.

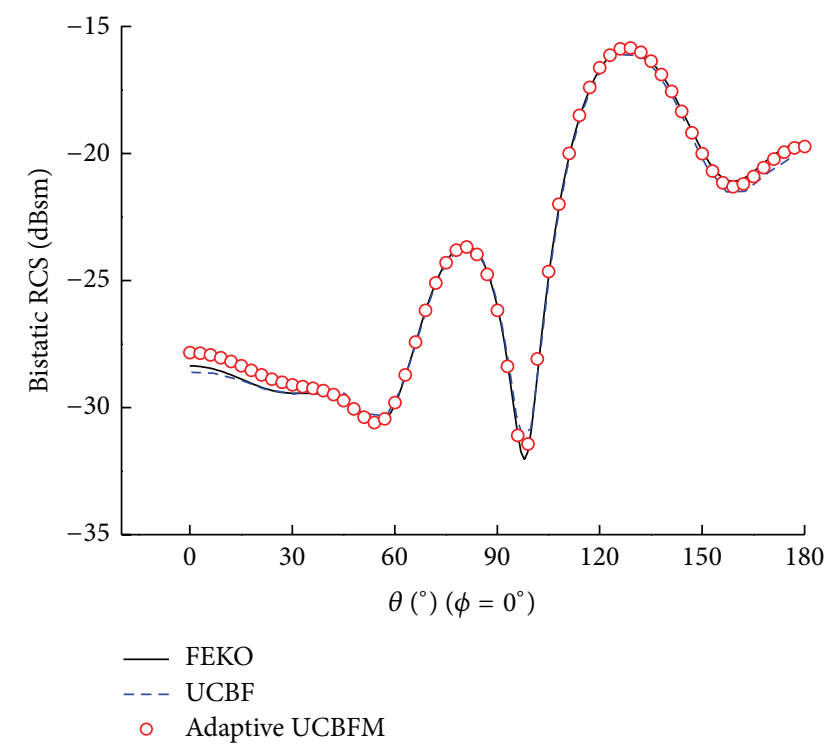

Figure 5: Bistatic RCS of PEC almond at $2.1 \mathrm{GHz}$.

UCBFM has compressed much more CPU time and random memory demand than conventional MoM and UCBF.

\section{Conclusion}

This paper has presented a method for analyzing broadband electromagnetic behaviors of metallic objects. The proposed technique is based on the adaptive construction of the UCBFs over a wide frequency band. This method leads to relatively small number of UCBFs at lower frequencies, so the reduced matrix size can be decreased at lower frequency band, which leads to a significant reduction of solver time with respect to conventional CBFM procedure. The results thus demonstrate that the proposed approach is able to accurately compute the RCS in arbitrary frequency band, resulting in a substantial time-saving, without a compromise of the accuracy.

\section{Conflict of Interests}

The authors declare that there is no conflict of interests regarding the publication of this paper. 


\section{Acknowledgments}

This work was supported by the National Natural Science Foundation of China under Grant no. 61172020 and the Specialized Research Fund for the Doctoral Program of Higher Education of China under Grant no. 20123401110006.

\section{References}

[1] G. J. Burke, E. K. Miller, S. Chakrabarti, and K. Demarest, "Using model-based parameter estimation to increase the efficiency of computing electromagnetic transfer functions," IEEE Transactions on Magnetics, vol. 25, no. 4, pp. 2807-2809, 1989.

[2] C. J. Reddy, M. D. Deshpande, C. R. Cockrell, and F. B. Beck, "Fast RCS computation over a frequency band using method of moments in conjunction with asymptotic waveform evaluation technique," IEEE Transactions on Antennas and Propagation, vol. 46, no. 8, pp. 1229-1233, 1998.

[3] X. C. Nie, N. Yuan, L. W. Li, and Y. Gan, "Fast analysis of RCS over a frequency band using pre-corrected FFT/AIM and asymptotic waveform evaluation technique," IEEE Transactions on Antennas and Propagation, vol. 56, no. 11, pp. 3526-3533, 2008.

[4] E. H. Newman, "Generation of wide-band data from the method of moments by interpolating the impedance matrix," IEEE Transactions on Antennas and Propagation, vol. 36, no. 12, pp. 1820-1824, 1988.

[5] E. Lucente, A. Monorchio, and R. Mittra, "Fast and efficient RCS computation over a wide frequency band using the "Universal" Characteristic Basis Functions (UCBFs)," in Proceedings of the IEEE Antennas and Propagation Society International Symposium, pp. 3580-3583, June 2007.

[6] M. de Gregorio, G. Tiberi, A. Monorchio, and R. Mittra, "Solution of wide band scattering problems using the characteristic basis function method," IET Microwaves, Antennas and Propagation, vol. 6, no. 1, pp. 60-66, 2012.

[7] M. Degiorgi, G. Tiberi, and A. Monorchio, "Wide band scattering through the use of the universal characteristic basis functions (UCBFs)," in Proceedings of the IEEE International Symposium Antennas and Propagation and CNC/USNC/URSI Radio Science Meeting, Toronto, Canada, July 2010.

[8] V. V. S. Prakash and R. Mittra, "Characteristic basis function method: a new technique for efficient solution of method of moments matrix equations," Microwave and Optical Technology Letters, vol. 36, no. 2, pp. 95-100, 2003.

[9] M. Degiorgi, G. Tiberi, A. Monarchic, G. Manara, and R. Mittra, "An SVD-based method for analyzing electromagnetic scattering from plates and faceted bodies using physical optics bases," in Proceedings of the IEEE Antennas and Propagation Society International Symposium, pp. 147-150, July 2005.

[10] S. M. Rao, D. R. Wilton, and A. W. Glisson, "Electromagnetic scattering by surfaces of arbitrary shape," IEEE Transactions on Antennas and Propagation, vol. 30, no. 3, pp. 409-418, 1982.

[11] A. C. Woo, H. T. G. Wang, M. J. Schuh et al., "Benchmark radar targets for the validation of computational electromagnetics programs," IEEE Antennas and Propagation Magazine, vol. 35, pp. 84-89, 1993. 

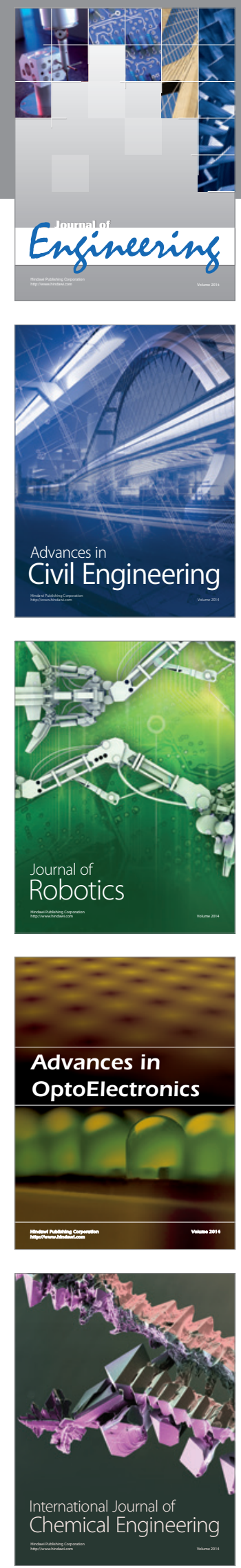

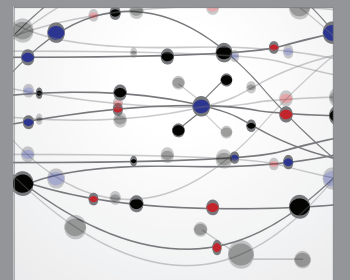

The Scientific World Journal
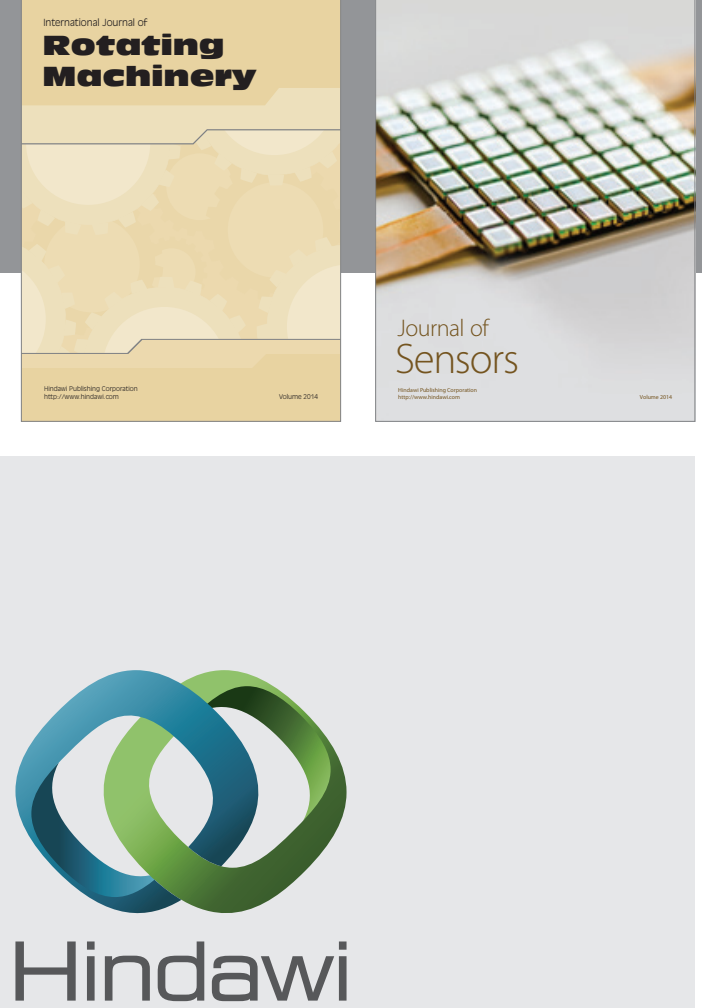

Submit your manuscripts at http://www.hindawi.com
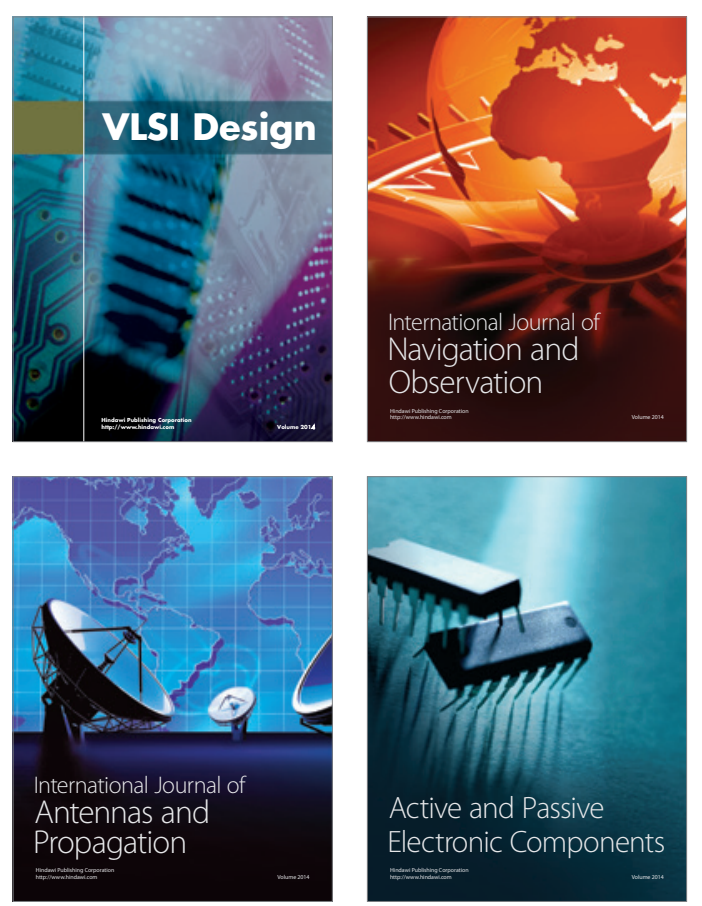
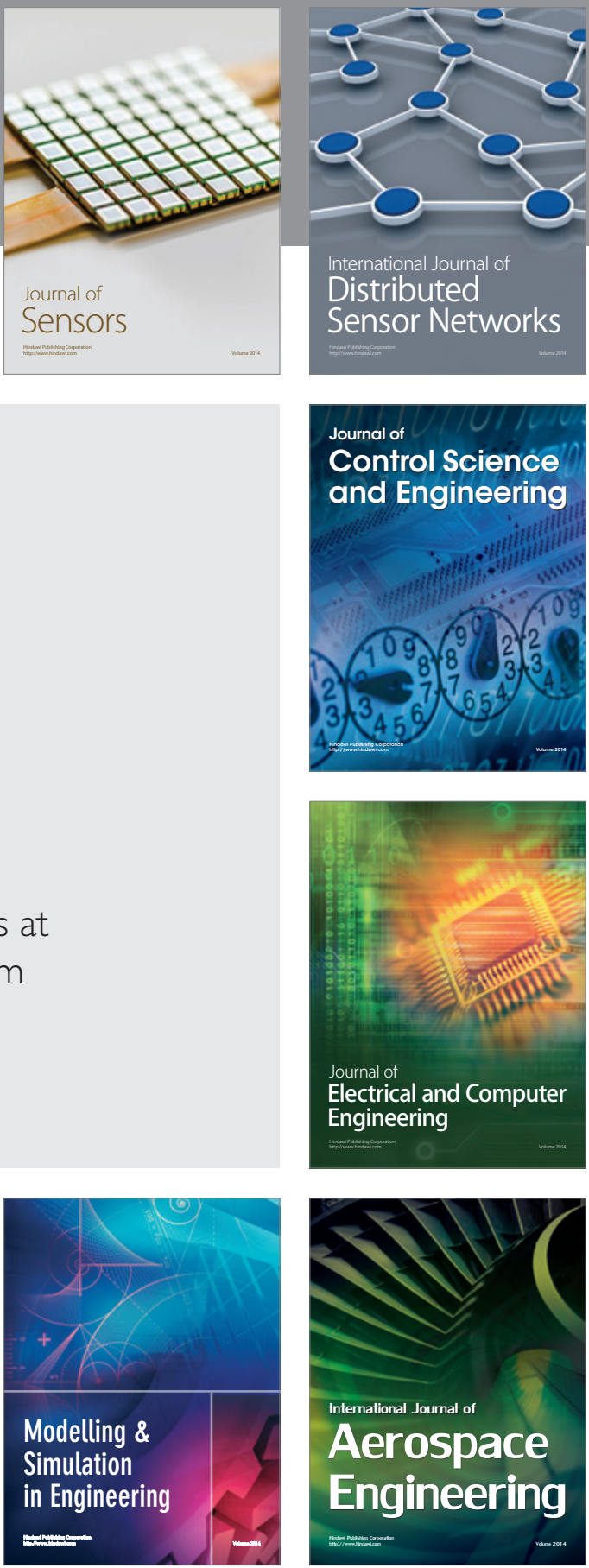

Journal of

Control Science

and Engineering
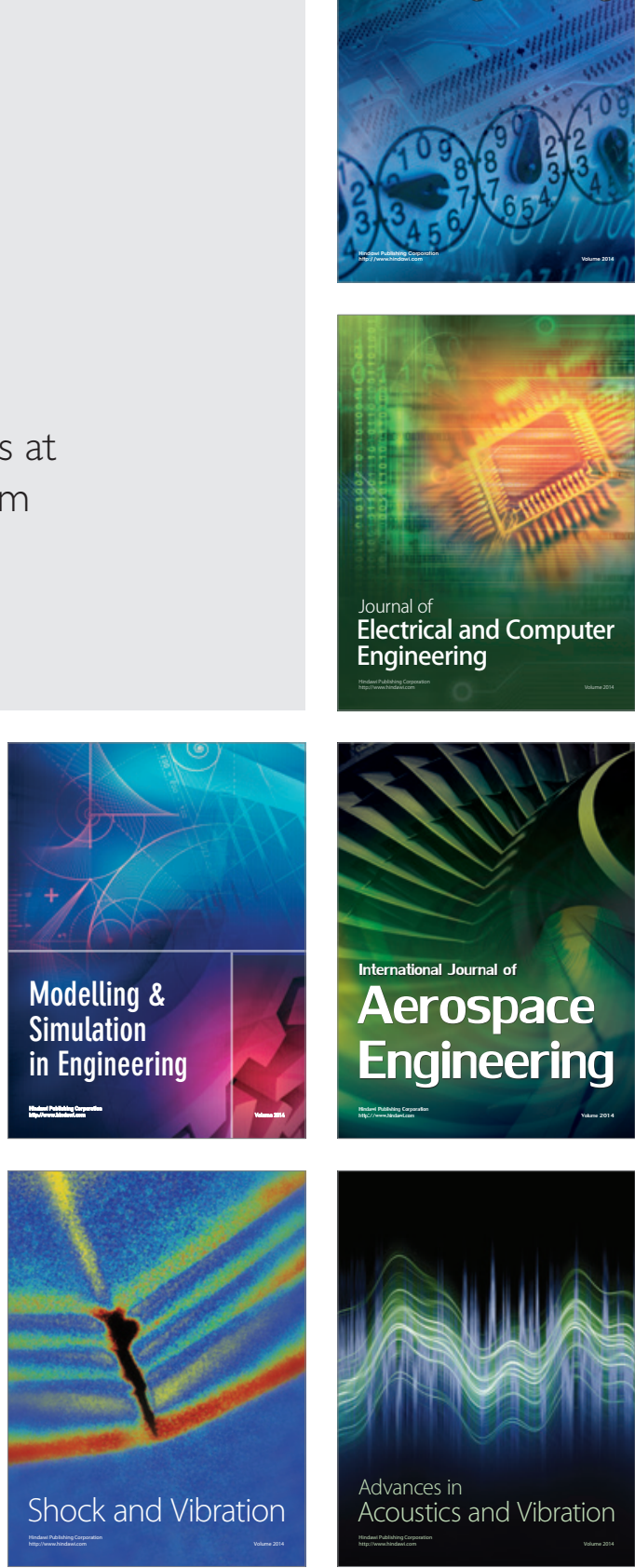